LA-7970-MS

Informal Report
Investigation of the Use of

Position Sensitive Detectors with a

Magnetic Spectrometer

U.

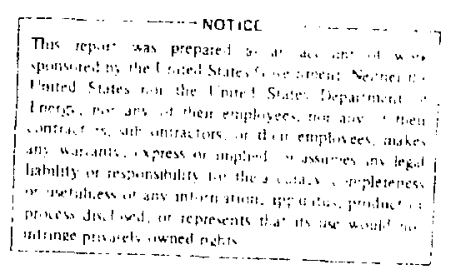

$\frac{7}{\frac{2}{0}}$ 


\title{
Investigation of the Use of \\ Position Sensitive Detectors with a \\ Magnetic Spectrometer
}

\author{
R. A. Olson
}

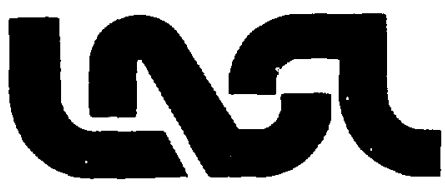




\title{
IN VEST IGATION OF THE USE OF POSITION SENSIT I VE DETECTORS
}

WITH A MAGNET IC SPECTROMETER

\author{
by
}

R. A. 01 son

\begin{abstract}
ABST RACT
A position sensitive detector is a solid-state sensor having unique properties which allow it to determine the position of the incident particle. An attempt is made to evaluate the usefulness of this type of detector in conjunction with a particle magnetic spectrometer.
\end{abstract}

JNT RODUCT ION

Solid state detectors have been around a relatively long time and have proven to be valuable tools for the detection of charged particles and the measurement of their energies. Their use in satellites and probes is widespread due to their convenient size and adaptability.

However, a development employed by Ortec, holds promise for increased use in spacecraft work. This consists of the addition of a resistive layer to the surface of the semi-conductor. Comparing a differential signal from the layer with the standard energy signal by using a special analyzing amplifier, a location may be derived indicating the incident position of the particles. This property of being "position sensitive" appears to be useful for certain applications .

Heretofore, magnetic spectrometers have had limited use in spacecraft work. However, with the coming availability of the Space Shuttle where large payloads are possible, a magnetic spectrometer of greater range seems feasible. Using a position sensitive detector in conjunction with a magnetic spectrometer seems to be a logical application of the device and a useful means of looking at energetic electrons. 
This report is the result of a study of the position sensitive detector and an attempt to evaluate its usefulness when employed with the spectrometer.

\section{DESCR IPT ION}

The Position Sensitive Detector (PSD) developed by Ortec is made of N-type silicon with boron ions implanted in the surface to provide a resistive layer. It is this uniform resistive layer that allows one to obtain the position information. The PSD can be treated as a solid-state delay line where the distributed capacitance of the depletion layer can be assigned to a series of resistors that constitute the resistive layer. Fig. 1.

The point of impact of an event on the surface of the PSD determines the proportion of pulse amplitudes that appear at connection points 1 and 2 . A pulse genarated at say point A will of course be smaller when measured at 1 than at 2 and it is precisely this difference that makes the device a Position Sensitive Device. One of these points is normally operated at ground with the other going to a charge-sensitive preamplifier and being regarded as the position output. The PSD is biased through connection 3 with this connection also providing an output for the energy absorbed by the detector. $\Lambda$ s employed in the laboratory, the system is as recommended by Ortec. Fig. 2.

The spectrometer consists simply of two flat, rectangular permanent magnets in a soft iron box. A schematic view of the spectrometer, with PSD in

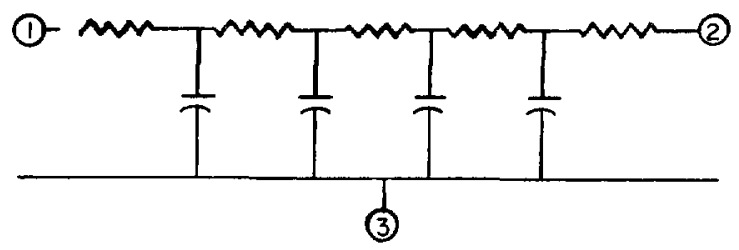

Fig. 1. Equivalent circuit of PSD.

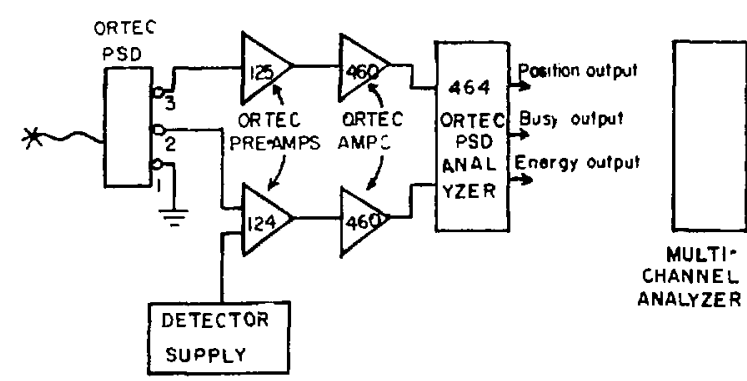

Fig. 2. PSD Operation set-up. 


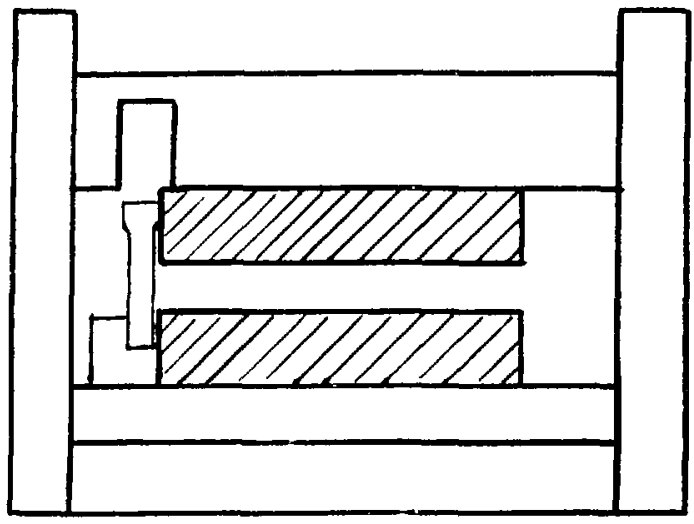

Fig. 3. Cross-sectional view of spectrometer.

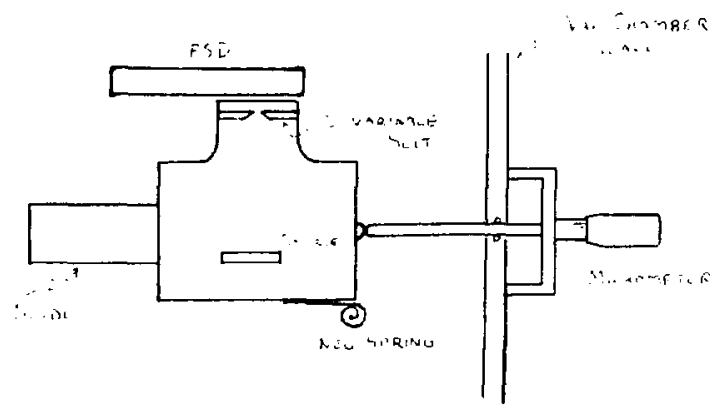

Fig. 4. Sketch of calibration set up.

place, is shown in Fig. 3. The magnets are approximately 3 inches by 6 inches and $9 / 16$ inch thick and are made of a cobalt alloy. The spacing between them is approximately 1 centimeter.

\section{PROCEDURE}

A determination of the linearity of the PSD was nade by using alpha particles from a ${ }^{239} \mathrm{Pu}$ source and a vernier locating device. This device consists of a small, raised table upon which is attached a spring-loaded sliding platform. The PSD used in this study is $45 \mathrm{~mm}$ long and $7 \mathrm{~mm}$ wide with a sensitive thickness of 1000 microns. The PSD was mounted on the fixed portion of the position locator and the source and a $0.5 \mathrm{~mm}$ slit were mounted on the movable section. The whole arrangement was then placed inside a vacuum shamber. The movable section with the source and slit could then be accurately positioned in front of the PSD from outside the vacuum chamber by a Iinear micrometer. A schematic drawing of the setup is shown in Fig. 4.

The magnetic field of the spectrometer was measured by means of a Universal Gaussmeter, model \#1965. An average value of 2500 gauss was obtained for the field this way and this provided information as to where the peaks should occur. The magnetic field can be assumed to be constant over the area of the poles and fringe effects ignored for the purpose of this investigation. 
The spectrometer with the PSD as the sensitive element was then calibrated using the internal conversion electrons from ${ }^{207} \mathrm{Bi}$. Again, the spectrometer and PSD were placed inside a vacuum chamber. Also an arrangement of two pulsers, one triggered by the other with one providing positive and the other providing negative pulses, was used to calibrate the electronics in relation to position and energy. The negative pulse was fed into the "energy" preamplifier at a value to simulate the high-energy peak and the positive signal entered the "position" preamplifier and was varied to simulate position pulses.

\section{RESURTS}

The calibration with the ${ }^{23}$ ipu alpha source revealed that the linearity of the detector with respect to position was surprisingly good. A plot of the data is shown in Fig. 5. The response to the pulser arrangement was quite linear also as is pictured in Fig. 6.

The position resolution determined at only one position gave a resolution of $0.76 \mathrm{~mm}$ as compared to $0.43 \mathrm{~mm}$ from the manufacturers spec sheet for this detector. However, the slit width for our experiment was $0.5 \mathrm{~mm}$ whereas the

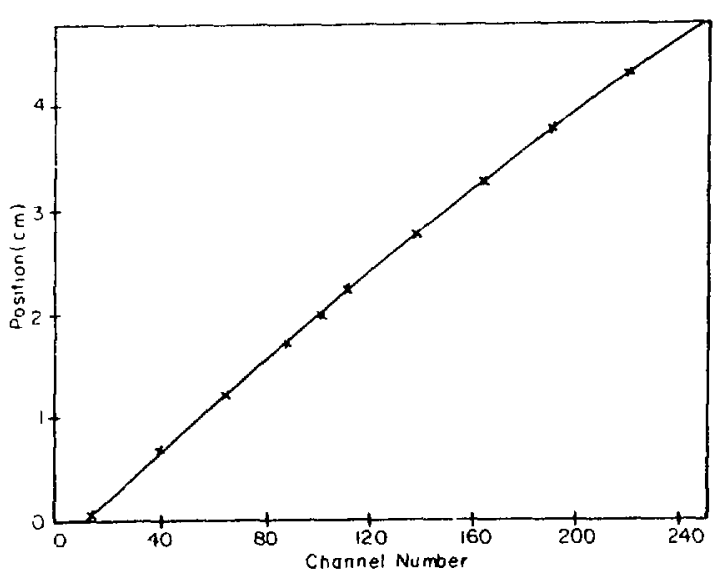

Fig. 5. Graph of linear resporise of PSD.

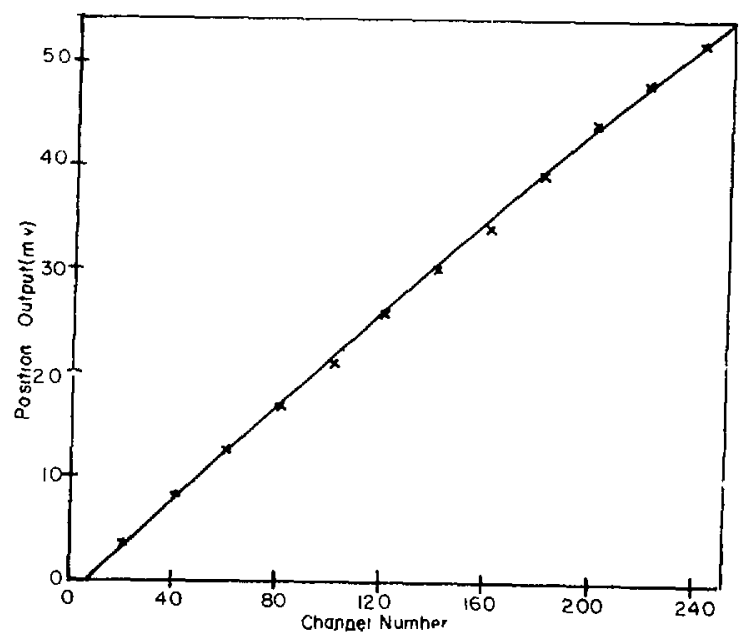

Fig. 6. Graph of response of a electronics. 
manufacturer's width was only $0.1 \mathrm{~mm}$. The source was approximately $4 \mathrm{~cm}$ from the PSD and the slit only $0.4 \mathrm{~cm}$ from the PSD. A worst case calculation gives divergence of $0.6 \mathrm{~mm}$ for the alpha particle beam. Considering this and assuming the manufacturer's specification is determined mainly by electronic noise, we get

$$
\sqrt{(0.43)^{2}+(0.5)^{2}}=.66 \mathrm{~mm}
$$

which is in fairly good agreement.

Ortec recommends that the PSD be used when analyzing particles with energies greater than $1 \mathrm{MeV}$. However. Etcheto et al.', have looked into their use at energies under $1 \mathrm{MeV}$ and they have found that the PSD is usable at lower energies but that the resolution deteriorates. This is due mainly to the effect of noises on the system. Johnson noise, introduced by the resistive layer, becomes important at low frequencies. The divided signals through the two preamplifiers are not independent and have an important effect on the correlated noise sources.

The field of the spectrometer as measured by the Universal Gaussmeter was 2500 gauss. Evaris ${ }^{2}$ (p. 894) Rives the following formula relating the kinetic energy of electrons in a magnetic field to their radius of curvature

$$
\left(\frac{E}{0.51098}+1\right)^{2}=\left(\frac{B p}{1704}\right)^{2}+1
$$

where $E=$ electron energy in MeV.

$$
\begin{aligned}
& B=\text { magnetic field in Gauss } \\
& \rho=\text { radius of curvature of electron in } \mathrm{cm} \\
& B \rho=\text { momentim in gauss-centimeters } \\
& 0.51098 \mathrm{MeV}=\mathrm{m}_{0} c^{2} \text {, rest energy of electron. }
\end{aligned}
$$

For a 1 MeV electron,

$$
\mathrm{B} p=4742 \text { gauss-centimeters }
$$

and for a field of 2500 gauss, as measured by the probe

$$
\rho=1.9 \mathrm{~cm}
$$

A position spectrum obtained lising ${ }^{207} \mathrm{Bi}$ internal conversion electrons directed into the magnetic spectrometer and with the PSD as the sensor is shown in Fig. 7 .

The only peak obtained is due to the high energy electrons. ${ }^{207} \mathrm{Bi}$ has two high energy electron Iines, one at $1.048 \mathrm{MeV}$ and the other at $0.976 \mathrm{MeV}$. With 


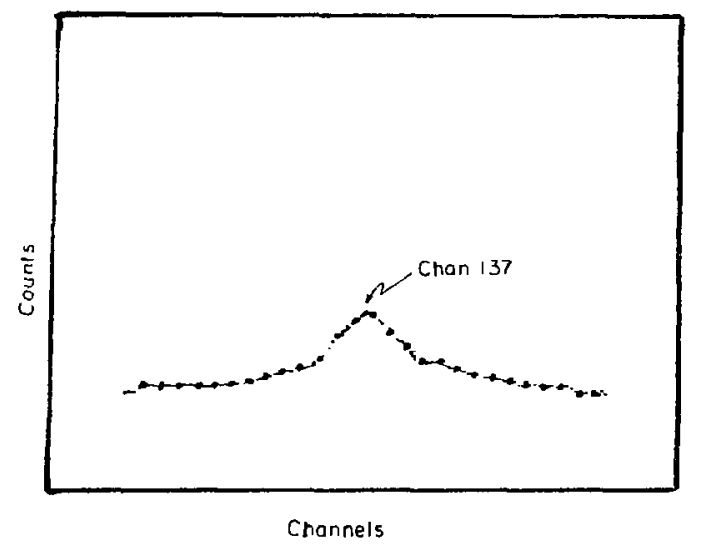

Fig. 7. Sketch of scipe trace of position spectrum.

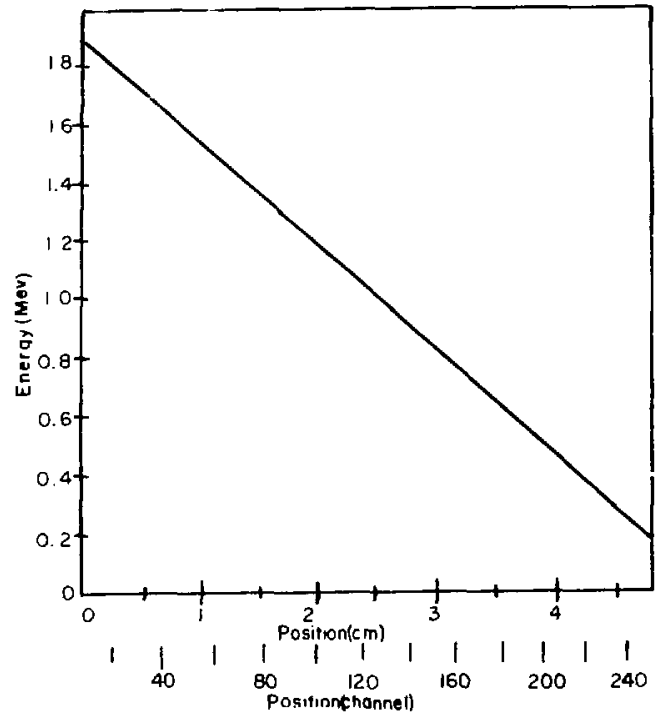

Fig. 8. Graph of energy of electrons vs position.

an energy resolution of $70 \mathrm{keV}$ and with all the other noise contributions, these two lines are not resolvable. However, the $480 \mathrm{keV}$ peak is not resolvable either. Allowing for the distance between the source and the active area of the PSD, $1.55 \mathrm{~cm}$, using 2500 gauss as measured by the fluxmeter for calculations, and referring to the linear response curve, Fig. 6, the 1 $\mathrm{MeV}$ electron peak should fall at approximately channel 122 and the $480 \mathrm{keV}$ peak should appear at approximately channel 200. With semi-circular spectrometers of the constant field type ${ }^{3}$, the central ray from the source always falls on the high-energy edge of the peak. Channel 122 is indeed on the high energy edge of the peak but there is no evidence of the $480 \mathrm{keV}$ peak at channel 200 although a slight hump can be seen at channel 180. However, this is understandable in that the intensity of this peak is quite low in comparison with the $1 \mathrm{MeV}$ peak ( $\mathrm{29 \%}$ ) and is probably hidden in the noise. The measured intrinsic noise of the sensor is $45 \mathrm{keV}$ and the total noise contribution from the sensor, preamplifier and amplifier combination is 50 kel or less.

Figure 8 is a graph relating energy of the analyzed electron to the position that it should fall on the PSD. 


\section{CONC LUS IONS}

The Position Sensitive Device is an interesting development in the solidstate sensor field. It obviously has its limitations, however. As employed here, the device does not appear very useful. It has been shown by others ${ }^{1}$ that PSDs can be used at low energies ( $s 200 \mathrm{keV}$ ), but the spectrometer itself limits the resolution of the sensor, contributing greatly to image spreading. Typically, a semi-circular spectrometer being a single-focusing device has relatively poor transmission, so a compromise must be reached between image size and counting rate. Magnetic fields may be treated as having optical properties and a formula applying this feature is given by Segre 4

$$
h^{\prime}=h+2 \pi a \theta
$$

where $h^{\prime}$ is the image height, $h$ is the height of the object, a is the radius of the semi-circular path, and $\theta$ is the half-angle of the apertire. The height of object is $0.3 \mathrm{~cm}$, the hal f-angle is $8.97^{\circ}$. and tr.; radius as previously calculated is $1.9 \mathrm{~cm}$ giving an image height of $2.21 \mathrm{~cm}$. This is greater than the magnet separation and would result in considerable scattering.

Again referring to Segre, a formula is found which calculates the width of the image or dispersion:

$$
S^{-}=s+a\left(\phi^{2}+\theta^{2}\right)
$$

where $S^{\circ}$ is the image width, $S$ is the source width, a is the radius of the semi-circular path, and $\phi$ and $\theta$ are the half-angles of the source. $\phi$ and $\theta$ are equal since the collimator is a circular hole which gives

$$
s^{\prime}=s+2 a \theta^{2}
$$

Using values obtained previously, the image width is found to be $0.4 \mathrm{~cm}$, an increase of only $0.1 \mathrm{~cm}$ on the source width.

There are many more contributions to the image spreading including source thickness and a relatively poor vacuum system. The vacuum chamber was normally operated at approximately $10^{-3}$ Torr which according to Madjanovic ${ }^{3}$ ( $p$. 
31) is not a good vacuum for electron transmission. He recomends at least $10^{-4}$ Torr. Hrwever, using an equation from sears $s^{5}$ for the electron mean free path, $\lambda_{\mathrm{e}}$

$$
\lambda_{e}=\frac{1}{\pi \rho^{2} n}
$$

where $\rho$ is the molecular radius and $n$ is the number of molecules per unit volume and using $\pi a$ for the path length of the electron approximately 0.24 collisions are found to occur at the pressure of $10^{-3}$ Torr. Also, it was found necessary to surround the spectrometer with considerable lead in order to cut down the background which obscured any peaks. This background appeared to be due to Bremsstrahlung and scattering from the chamber and the spectrometer itself.

Considering all these things, it does not appear that the full potential of the position sensitive device was attained. Lack of time and other suitable electron sources prevented checking the PSD in more detail. Perhaps, by taking nore pains with the source (in particular by collimating it better), by using a better vacuum system and using a baffle arrangement inside the spectrometer, an increase in the resolution of the device can be realized. Also, it might be worthwhile to try a proportional counter in conjunction with the spectrometer. It would offer faster counting with a reduction in background and possibly better resolution.

\section{ACKNOWLEDGMENT S}

This work was dore under the auspices of the U.S. Department of Energy. The author wishes to thank P. R. Higbie for his advice and encouragement.

\section{REFERENCES}

1. J. Etcheto, J. Etcheto, R. Gendrin and B. De La Porte Des Vaux, Use of Position Sensitive Detectors at Energies Below 1 MeV. Nuc. Inst. and Methods, 108 (1973) 271-277.

2. R. D. Evans, The Atomic Nucleus (McGraw-Hill Book Co., New York, U.S.A., 1955). 
3. M. Madjenovic, Development of Magnetic B-Ray Spectroscopy, Lecture Notes in Physics (Springer-Verlag. New York, U.S.A., 1976).

4. E. Segre, Experimental Muclear Physics, Volume III (John Wiley \& Sons, Inc., New York, 1959).

5. F. W. Sears, Thermodynamics (Addison-Wesley Publishing Co.., Inc., Cambridge 42, MA, 1955). 